\title{
BMJ Open Codesigning a mobile gamified attention bias modification intervention: research protocol
}

\author{
Melvyn WB Zhang, ${ }^{1}$ Sandor Heng, ${ }^{1}$ Guo Song, ${ }^{1}$ Daniel Shuen Sheng Fung, ${ }^{2}$ \\ Helen Smith ${ }^{3}$
}

To cite: Zhang MWB, Heng S, Song $\mathrm{G}$, et al. Codesigning a mobile gamified attention bias modification intervention: research protocol. BMJ Open 2019;9:e031897. doi:10.1136/ bmjopen-2019-031897

- Prepublication history and additional material for this paper are available online. To view these files, please visit the journal online (http://dx.doi. org/10.1136/bmjopen-2019031897).

Received 28 May 2019 Revised 06 September 2019 Accepted 23 September 2019

Check for updates

(c) Author(s) (or their employer(s)) 2019. Re-use permitted under CC BY-NC. No commercial re-use. See rights and permissions. Published by BMJ.

\section{${ }^{1}$ National Addictions}

Management Service, Institute of Mental Health, Singapore,

Singapore

${ }^{2}$ Department of Developmental Psychiatry, Institute of Mental Health, Singapore, Singapore ${ }^{3}$ Family Medicine and Primary Care, Lee Kong Chian School of Medicine, Nanyang Technological University, Singapore, Singapore

Correspondence to Dr Melvyn WB Zhang; melvynzhangweibin@gmail.com

\section{ABSTRACT}

Introduction Advances in experimental psychology has highlighted the need to modify underlying automatic cognitive biases, such as attentional biases. While prior research has documented the effectiveness of cognitive bias modification, such interventions tend to be highly repetitive and individuals lack motivation to train over time. Participatory action research methods have been more widely applied in psychiatry to help design interventions that are of relevant to key stakeholders and end users. This study aimed to involve both healthcare professionals and patients in the joint codesign of a gamified mobile attention bias modification intervention.

Methods and analysis The participatory design research method adopted is that of a use-oriented design approach, in the form of a future workshop. 20 participants, comprising 10 healthcare professionals, 5 inpatients and 5 outpatients will be recruited to participate in three separate codesign workshops. In the first phase of the workshop, the participants share their critique of an attention bias modification intervention. In the second phase of the workshop, participants are asked to brainstorm features. The participants are also shown gamification approaches and are asked to consider if gaming elements could enhance the existing application. In the last phase, the participants are asked to sketch a new prototype.

Ethics and dissemination Ethical approval has been obtained from the National Healthcare Group's Domain Specific Research Board (approval number 2018/01363). The findings arising from this study will be disseminated by means of conferences and publications.

\section{INTRODUCTION}

Alcohol and illicit substances like cannabis, opioids and stimulants are highly abused globally. ${ }^{1}$ Illicit substance abuse and dependence is, in turn, associated with significant morbidity, such as that of retroviral diseases and mortality. ${ }^{1}$ The harmful use of alcohol has resulted in an overall mortality of three million individuals in 2016. ${ }^{2}$ Pharmacological treatment of substance use disorder is limited, ${ }^{3}$ as there remain no approved medications for cannabis use and stimulant use disorders. Symptomatic medications are typically used

\section{Strengths and limitations of this study}

- Exploring participatory design research methods to codesign an application that is relevant and accepted by patients.

- Methods are based on a user-centred approach.

- The views of academics and healthcare professionals are also considered in the design process.

- A diverse group of patients at varying stages of addiction are included.

- Due to potential hierarchy issues, it is not possible to have healthcare professionals and patients in the same workshop, as it might limit sharing of perspectives.

in the treatment of the acute intoxication symptoms, and psychological-based therapies have the mainstay in the maintenance of abstinence among individuals. Psychological therapies, such as that of cognitive-behavioural therapy, have been used, but its effectiveness remains unclear, with some studies reporting that at least $40 \%-50 \%$ of individuals relapse after a year and $70 \%$ relapse within 3 years. ${ }^{4}$ Conventional psychotherapies might not have addressed all the factors leading an individual to a slip or relapse back into his addictive disorder. The advances in experimental psychology have led to the discovery of the dual-process theoretical model,${ }^{56}$ which seeks to explain why relapses are common despite having individuals undergoing moderately effective therapies like cognitive-behavioural therapy. Psychotherapies like cognitivebehavioural therapies have addressed only the conscious cognitive control processes and not the underlying automatic, unconscious processes. Based on the dual-process theory, the continuous usage of a substance thus results in increased appetitive processes and the corresponding weakening of executive control. ${ }^{7}$ Motivation in regulating these appetitive processes is also diminished. ${ }^{7}$ 
Two common automatic processes among individuals with addictive disorders are that of attention and approach biases. Attention biases refer to the preferential allocation of attentional processes towards substance-related cues, ${ }^{5}$ whereas approach biases refer to the automated tendencies for individuals to seek out and reach for substance-related stimuli. ${ }^{8}$ To date, the advances in experimental psychology have led to there being both direct and indirect measures of attention biases, in order to ascertain whether an individual has any underlying attentional biases. Indirect measures, such as the Stroop $\operatorname{task}^{9}$ and the Visual Probe task, are typically used..$^{10}$ These tasks (Stroop and visual probe) could also be used for the modification of biases, ${ }^{11} 12$ which implies that these automatic processes are being retrained, such that the attention is being directed away from the stimuli of interest. The effectiveness of bias modification has been extensively studied. Cristea $e t a l,{ }^{13}$ in their prior review, reported that cognitive bias modification for participants with alcohol and tobacco use disorders was moderately effective (Hedges' G that of 0.60). Other studies conducted among a clinical cohort have similarly reported that bias modification helped in the reduction of biases and led to various positive outcomes. ${ }^{1415}$

Up until recently, most of these interventions are delivered within the confines of the laboratory. ${ }^{16}$ Delivering bias modification interventions within the confines of a laboratory has its intrinsic advantages, as it allows for better supervision and compliance with the task. ${ }^{16}$ Undertaking such an intervention is, however, tedious, given that participants routinely must complete numerous repeats of the same task. Technological advances in the past decade have transformed how these tasks are delivered. To date, there are both web versions of bias modification interventions ${ }^{17}$ and mobile versions. ${ }^{18}$ Zhang et al ${ }^{18}$ in their recent review, highlighted eight published studies involving a mobile bias modification tool, and of the eight studies, seven reported the mobile intervention to be effective in modifying both biases and underlying behaviour. While technological advances have allowed for increased accessibility, there remained some studies indicating that there was no effectiveness. ${ }^{19}$ In the prior review, one study involving participants with social anxiety disorder reported bias modification to be ineffective. Notably, there were three other studies involving participants with social anxiety disorder reporting positive results, and all these studies have included gamification strategies. In recent years, there has been an increasing awareness that gamification could potentially engage patient's engagement. Gamification could increase the intrinsic motivation to train, as bias modification interventions tend to be repetitive. ${ }^{19}$ Gamified bias interventions were only effective in $50 \%$ of the identified studies. ${ }^{20}$ In the prior review, gaming elements that were applied included that of animations, sounds, feedback and a point-scoring system ${ }^{20}$; such strategies were considered, given that they could potentially increase engagement and motivation. Hoffmann $e t a l,{ }^{21}$ in their review of gamification strategies applied in stress management applications, have formulated a taxonomy of gaming approaches, that of economic, social, performance and embedding-focused strategies. Similarly, each of these strategies could help in user's engagement and motivation.

Participatory action research methods are instrumental in developing an application that is relevant for the end user. Participatory action research refers to a process of 'systematic inquiry, with the participation of those affected by the problem being studied, for education and action or effecting social change'.$^{22}$ Thus, participatory action research typically includes key stakeholders in the research process, in order to better the research and to ensure the generalisability of the findings. Such form of research has since been widely applied in medicine and psychiatry. ${ }^{23}$ Zhang $e t a l^{23}$ reported there were at least seven studies that have to describe how these methods have been applied for technological interventions in psychiatry. Of the articles identified previously, the psychiatric conditions in which participatory action research has been applied were those of perinatal depression, dementia, self-harm, and general mental health or youth mental health issues. ${ }^{23}$ The most common methods of participatory action research used have been those of cocreation workshops and focus groups. ${ }^{23}$ Zhang $e t a l^{23}$ highlighted the need to apply these methods for bias modification intervention research. Such methods help explore perceptions about the tasks and could help in the refinement of the existing task, and these methods could help researchers to understand reasons underlying the diminishing intrinsic motivation and interest in such interventions and methods that could be applied to help increase motivation to train. Moreover, Zhang $e t a l^{24}$ also reported there were at least 17 bias modification applications on the commercial store, with only one application that has had an academic input. It is evident from the review that there is a great divide between the academics, developers and the end users (or patients) themselves. Including methods of participatory design research could thus help to bridge this existing disconnect between the different stakeholders.

The aim of the current research was to involve both healthcare professionals and patients in the codesign of a gamified mobile attention bias modification intervention. The codesign processes have been focused on the most fundamental of all the cognitive biases, that of attention biases. Approach biases are more complicated in retraining as compared with attention biases, as they involve not only attention but also the activation of the relevant behavioural schemata. This research aims to answer the following key questions: (1) what are the perspectives of healthcare professionals and patients about a mobile attention bias modification intervention? (2) what features would healthcare professionals and patients prefer in a mobile attention bias modification intervention, to help minimise attrition from task and to increase both intrinsic and extrinsic motivations in completing the intervention? (3) would gamification (ie, 
the application of gaming elements) help in the enhancement of the existing mobile bias intervention task? What gaming elements are preferred?

\section{Design approach}

This study uses the principles of participatory action research, the mode of research that uses 'systematic inquiry, with the participation of those affected by the problem being studied, for education and action or effecting social change'. ${ }^{25}$ There are several modes of participatory action research, including Software Technology for Evolutionary Participatory Systems Development, MUST (Danish acronym for theories and methods of initial analysis and design activities) and Cooperative Experimental System Developmental Approach. ${ }^{25}$ We have chosen to adopt a use-oriented design, ${ }^{25}$ which involves there being a real-life problem, understanding practice, identifying needs and wishes, describing requirements, concretising and materialising, and lastly testing and evaluation. In particular, the codesign workshop currently proposed, based on the use-oriented design approach is like that of a future workshop, which was first introduced in 1987 by Jungk and Müllert. ${ }^{26}$ The use-oriented design approach has been selected as it is explorative and allows for there to be negotiation and collaboration on the design, before a stepwise refinement through sketching and prototyping and is thus most appropriate for us in addressing our research question.

As our goal was the codesign and codevelopment of a new gamified novel attention bias modification interventions, we will use future workshops as these are optimal for generating new and innovative ideas. Traditionally, a future workshop involves three key phases. In the first phase, individuals are asked to point out their critiques to a known problem. In the second phase, individuals are asked to brainstorm for positive, and at times, even impossible solutions to the same problem. In the third phase, participants are then asked to transform their proposed ideas into something concrete and achievable. ${ }^{27}$

We propose a total of three codesign workshops, one of which will involve healthcare professionals, and the other two, patients, who are recruited both from the inpatient unit and from the outpatient settings. It was our preference for healthcare professionals and patients to jointly participate in the same workshop, but our ethics approving authority did not recommend this. They were concerned that the mix of healthcare professionals and patients might result in diminished participation from patients. We were unable to have both groups of patients attending the same workshop, mainly due to logistical reasons.

\section{Study settings and recruitment}

The current study will be conducted at the National Addictions Management Service (NAMS), Institute of Mental Health. NAMS is the largest addiction treatment centre in Singapore, providing treatment for both substance and behavioural addictions. Healthcare professionals will include addiction psychiatrists, addiction trained psychologist and counsellors. Two distinct groups of patients will be invited to participate in the codesign workshop, to represent patients of different stages of recovery. There will be a group of patients who have been attending regular evening groups at the NAMS. These patients are clinically stable and mostly abstinent from substance use. The other group will be inpatients who are currently undergoing detoxification or rehabilitation of their substance use.

\section{Participants}

A total of 20 participants will be recruited. Of the 20 participants recruited, 10 will be patients ( 5 inpatients and 5 outpatients), and the remaining 10 will comprise healthcare professionals. Patients will be included in the study if they (1) are aged between 21 and 65 years old; (2) are diagnosed with a primary psychiatric disorder of alcohol, opioid, cannabis and stimulant use disorder; (3) are able to speak and write in English; and (4) know how to use a smartphone device. For healthcare professionals, they must be currently working in an addiction unit, actively involved in the treatment of individuals with addictive disorders and must have a minimum of 2 years of experience working with clients with addictive disorders.

Patients will be excluded from the study if they (1) have a significant psychiatric comorbidity (moderate to severe depressive disorder, anxiety disorder and psychotic disorder); (2) are non-English-speaking; or (3) have an existing cognitive impairment or intellectual disability.

\section{Workshop procedures}

All participants will be asked to complete a questionnaire prior to participating in the codesign workshop. For patients, the demographic and clinical questionnaire will collate their baseline demographic information, their main drug of abuse, the total duration of usage and the duration in which they have been abstinent from the drug. In addition, for patients recruited from the inpatient unit, they will have to indicate whether they are in the detoxification or rehabilitation phase of treatment, and the number of days they have been on the ward. All healthcare professionals will have to complete a demographic questionnaire and about their experience in addiction work. The questionnaires that are used in the study are included in online supplementary appendix 1. The questionnaire for patients consists of 16 questions, and that for healthcare professionals consists of 5 questions.

In the current protocol, we have intentionally described in more detail the conduct of the workshop and how we will be engaging the relevant stakeholders. The objective of this is to allow for future replicability of our methods.

\section{Phase I}

In accordance to the guiding principles as aforementioned, participants are first told of the rationale of the research project and the objectives of the study. 
Participants will then be shown a presentation of an existing mobile attention bias modification application.

They will also be able to try the application on the provided tablet devices. Participants are then asked individually to narrate their perspectives and specifically their critiques of the existing application. If they are uncomfortable with sharing, all participants are offered note pads for them to document down their perspectives. Participants are allowed 30-40 min to complete phase I.

The following questions will be asked:

1. Having seen and used the existing application, what are your thoughts about it?

2. What are some of the limitations of the current applications?

\section{Phase II}

Participants will then be asked to brainstorm features that could be added to the existing mobile application. The following questions will be asked, and participants will be encouraged to write down their ideas on sticky notes, following which these notes will be collated by members of the study team and discussed in the workshop.

1. What additional features do you think could be added to the application?
2. Why do you think that these additional features will be helpful?

As one of the objectives is to determine whether gamification could address some of the limitations of the existing application, participants will be shown screenshot examples of some of the attention bias commercial applications, which included elements of gamification. The applications that have been identified for use are based on Zhang et al. ${ }^{23}$ (2018) previous review of commercial bias modification applications. In addition, the facilitator of the workshop will also explain verbally some of the other common gamification techniques that have been previously published in the literature (table 1). They are as follows.

Participants will then be asked to narrate their perspectives about the gamification ideas shared. Participants are to individually select their top three gamification techniques that they feel are most appropriate to be applied in the existing application.

Participants are allowed 1 hour for this phase of the workshop.

\section{Phase III}

In the last stage of the workshop, participants are then divided into groups to come up with frame-by-frame

Table 1 Overview of gamification approaches (Hoffmann et $\mathrm{al}^{21}$ )

\section{Gaming approach}

Economic gamification techniques

1. Marketplace and economies

2. Digital rewards

3. Real-world prizes

\section{Description}

Providing gamers with a virtual currency that allows them to deal in game.

These include badges, game currency, game points, virtual goods and powers or abilities.

Provides gamers with options to exchange in-game credits for real-world prizes, such as vouchers or other forms of goods and services.

Social gamification techniques

1. Avatar

2. Agent

3. Competition

4. Teams

5. Parallel communication systems

6. Social pressure

Allows an individual to choose a virtual character to represent oneself.

A virtual character that helps, guides or provides instructions to the user.

Allow individuals to compete with other players or with each other.

Game that involves several individual players, allowing them to interact and form relationships.

Allows individuals to communicate with one another.

Ability of game to pressurise individuals to perform

a certain task so that he or she will be invited to subsequent events.
Performance-oriented
1. Feedback
2. Levels
3. Secondary game objectives
4. Ranks of achievement
5. Leaderboards
6. Time pressure

Embedding-focused

1. Narrative context

2. 3-D environment

3-D, three-dimensional.

\section{Spoken, visual or auditory feedback about user's} performance.

Information on the stage of a game one has attained. Secondary goals that reward the player on completion. Measurement of character development.

Allows for comparisons with other players. Predetermined time limits for task completion.

A storyboard or stories that guide the development of the character.

3-D models of objects that parallel the real world. 
sketches of a prototype application that incorporates the solutions that they have proposed. Participants can sketch freely on the paper provided, using any of the writing instruments. They are told that they are to include the original task but could modify it. For healthcare professionals, they will be divided into two groups of five participants each. For both the inpatient and outpatient groups, they will be divided into two groups of two and three participants each. All the groups are given 15 min to work on this task.

Prior to the completion of the workshop, participants will also be shown the set of substance images that have been incorporated into the existing application. Participants are to rate, using a Likert scale (with scores ranging from 1 to 10), how relevant the images are for an individual with an addictive disorder. Participants will also be asked to share their perceptions with the facilitator of the workshop. Participants are allowed $30 \mathrm{~min}$ to rate and share their perceptions.

\section{Data analyses}

The workshops will be audio recorded and transcribed verbatim subsequently. The principal investigator (MWBZ) will listen to the audio recordings of the workshop and develop a coding frame. Two separate researchers will also review and code the transcripts. The codes will then be reorganised into themes. NVivo V.12.0 will be used in the analysis.

\section{Data management and monitoring}

No participant-related identifiers will be captured on the hard copy questionnaires. All the completed hard copy forms and the informed consent forms will be stored in a secured facility, under lock and key. Descriptive statistical analyses (means and SD) will be performed on the quantitative data acquired. The audio recordings of the workshop will be transferred onto a local secured computer for storage, and the recording will also be removed from the recording device. The password of the local computer will be changed frequently, and only the principal investigator will have access to the local computer. All the records and audio recordings will be kept for 6 years after the completion of the study.

\section{Adverse events}

Any adverse events that occur during the conduct of the study will be reported to the Domain Specific Research Board (DSRB) according to the local institutional policy.

\section{Patients and public involvement}

This study will involve both patients and healthcare professionals in the codesign of an application that will be of relevance to their needs.

\section{Ethics and dissemination}

This study has obtained ethical approval from the National Healthcare Group's DSRB (approval number that of 2018/01363). We are in the process of recruitment and conducting the workshops. We expect the study to be completed within 1 year. The results of this study will be disseminated using conferences, both local and international, and publications in journals.

Contributors MWBZ, SH, GS, DSSF and HS jointly conceptualised and designed the study protocol. MWBZ wrote the initial protocol. SH, GS, DSSF and HS amended the initial protocol. All authors read and approved of the protocol prior to submission.

Funding MWBZ is supported by a grant under the Singapore Ministry of Health's National Medical Research Council (grant number NMRC/Fellowship/0048/2017) for $\mathrm{PhD}$ training. The funding source was not involved in any part of this project. This study has received funding from the National Healthcare Group and Lee Kong Chian School of Medicine, ALIVE (Games for Health Innovation Centre) grant.

Competing interests None declared.

Patient consent for publication Not required.

Provenance and peer review Not commissioned; externally peer reviewed.

Open access This is an open access article distributed in accordance with the Creative Commons Attribution Non Commercial (CC BY-NC 4.0) license, which permits others to distribute, remix, adapt, build upon this work non-commercially, and license their derivative works on different terms, provided the original work is properly cited, appropriate credit is given, any changes made indicated, and the use is non-commercial. See: http://creativecommons.org/licenses/by-nc/4.0/.

\section{REFERENCES}

1 Cristea IA, Kok RN, Cuijpers P. The effectiveness of cognitive bias modification interventions for substance addictions: a meta-analysis. PLoS One 2019;11:e0162226.

2 World Health Organization. Alcohol use disorders. extracted from. Available: http://www.who.int/substance_abuse/publications/global_ alcohol_report/en/ [Accessed last assessed 5th September 2019].

3 O'Brien CP. \& J. Mckay. Psychopharmacological Treatments for Substance Use Disorders in a Guide to Treatments that Work. United Kingdom: Oxford University Press, 2007.

4 Cutler RB, Fishbain DA. Are alcoholism treatments effective? the project match data. BMC Public Health 2005;5:75.

5 Field M, Cox WM. Attentional bias in addictive behaviors: a review of its development, causes, and consequences. Drug Alcohol Depend 2008;97:1-20.

6 Cristea IA, Kok RN, Cuijpers P. Efficacy of cognitive bias modification interventions in anxiety and depression: meta-analysis. $\mathrm{Br} \mathrm{J}$ Psychiatry 2015;206:7-16.

7 Stacy AW, Wiers RW. Implicit cognition and addiction: a tool for explaining paradoxical behavior. Annu Rev Clin Psychol 2010;6:551-75. Vol.

8 Boffo M, Smits R, Salmon JP, et al. Luck, come here! automatic approach tendencies toward gambling cues in moderate- to high-risk gamblers. Addiction 2018;113:289-98.

9 Ridley J. "Studies of interference in serial verbal reactions". J Experiment Psychol 1935;18:643-62.

10 MacLeod C, Mathews A, Tata P. Attentional bias in emotional disorders. J Abnorm Psychol 1986;95:15-20.

11 Christiansen P, Schoenmakers TM, Field M. Less than meets the eye: Reappraising the clinical relevance of attentional bias in addiction. Addict Behav 2015;44:43-50.

12 Zhang MW, Ying JB, Song G, et al. Recent advances in attention bias modification for substance addictions. PLoS One 2018;15.

13 Cristea IA, Kok RN, Cuijpers P. The effectiveness of cognitive bias modification interventions for substance addictions: a meta-analysis. PLoS One 2016;11:e0162226.

14 Manning V, Staiger PK, Hall K, et al. Cognitive Bias Modification Training During Inpatient Alcohol Detoxification Reduces Early Relapse: A Randomized Controlled Trial. Alcohol Clin Exp Res 2016;40:2011-9.

15 Schoenmakers TM, de Bruin M, Lux IFM, et al. Clinical effectiveness of attentional bias modification training in abstinent alcoholic patients. Drug Alcohol Depend 2010;109:30-6.

16 Jones EB, Sharpe L. Cognitive bias modification: a review of metaanalyses. J Affect Disord 2017;223:175-83.

17 Wittekind CE, Feist A, Schneider BC, et al. The approach-avoidance task as an online intervention in cigarette smoking: a pilot study. $J$ Behav Ther Exp Psychiatry 2015;46:115-20.

18 Zhang M, Ying J, Song G, et al. Attention and cognitive bias modification Apps: review of the literature and of commercially available Apps. JMIR mHealth and uHealth 2018;6:e10034. 
19 Beard C, Weisberg RB. Socially anxious primary care patients' attitudes toward cognitive bias modification (CBM): a qualitative study behavioural and cognitive psychotherapy 2012;40:618-33.

20 Zhang M, Ying J, Song G, et al. Gamified cognitive bias modification interventions for psychiatric disorders: review. JMIR Ment Health 2018;5:e11640.

21 Hoffmann A, Christmann CA, Bleser G. Gamification in stress management Apps: a critical APP review. JMIR Serious Games 2017;5:e13.

22 Green LW, George MA, Daniel M, et al. Study of participatory research in health promotion: review and recommendations for the development of participatory research in health promotion in Canada. Vancouer, BC, USA: BCRoyal Society of Canada, 1995.
23 Zhang MWB, Ying JB, Song G, et al. A review of gamification approaches in commercial cognitive bias modification gaming applications. THC 2018;26:933-44.

24 Zhang M, Ying J. Incorporating participatory action research in attention bias modification interventions for addictive disorders: perspectives. Int J Environ Res Public Health 2019;16:822.

25 Simonsen J, Robertson T, eds. Routledge International Handbook of Participatory Design. New York: Routledge, 2012.

26 Jungk R, Müllert N. Future workshops: how to create desirable futures. London, England: Institute for Social Inventions, 1987.

27 Brandt E, Binder T, Sanders EBN. Tools and techniques: ways to engage telling, making and enacting. In: Simonsen J, Robertson T, eds. Routledge international Handbook of participatory design. New York: Routledge, 2013: 145-81. 